\title{
Heavy fermion and Kondo lattice behavior in the itinerant ferromagnet $\mathrm{CeCrGe}_{3}$
}

\author{
Debarchan Das, ${ }^{1}$ T. Gruner, ${ }^{2}$ H. Pfau,${ }^{2}$ U. B. Paramanik,,${ }^{1}$ U. Burkhardt,${ }^{2}$ C. Geibel,${ }^{2}$ and Z. Hossain ${ }^{1,2, *}$ \\ ${ }^{1}$ Department of Physics, Indian Institute of Technology, Kanpur 208016, India \\ ${ }^{2}$ Max-Planck Institute for Chemical Physics of Solids, 01187 Dresden, Germany
}

(Dated: July 23, 2021)

\begin{abstract}
Physical properties of polycrystalline $\mathrm{CeCrGe}_{3}$ and $\mathrm{LaCrGe}_{3}$ have been investigated by x-ray absorption spectroscopy, magnetic susceptibility $\chi(T)$, isothermal magnetization $\mathrm{M}(\mathrm{H})$, electrical resistivity $\rho(T)$, specific heat $\mathrm{C}(T)$ and thermoelectric power $\mathrm{S}(T)$ measurements. These compounds are found to crystallize in the hexagonal perovskite structure (space group $P 6_{3} / m m c$ ), as previously reported. The $\rho(T), \chi(T)$ and $\mathrm{C}(T)$ data confirm the bulk ferromagnetic ordering of itinerant $\mathrm{Cr}$ moments in $\mathrm{LaCrGe}_{3}$ and $\mathrm{CeCrGe}_{3}$ with $T_{C}=90 \mathrm{~K}$ and $70 \mathrm{~K}$ respectively. In addition a weak anomaly is also observed near $3 \mathrm{~K}$ in the $\mathrm{C}(T)$ data of $\mathrm{CeCrGe}_{3}$. The $\mathrm{T}$ dependences of $\rho$ and finite values of Sommerfeld coefficient $\gamma$ obtained from the specific heat measurements confirm that both the compounds are of metallic character. Further, the $T$ dependence of $\rho$ of $\mathrm{CeCrGe}_{3}$ reflects a Kondo lattice behavior. An enhanced $\gamma$ of $130 \mathrm{~mJ} / \mathrm{mol} \mathrm{K}^{2}$ together with the Kondo lattice behavior inferred from the $\rho(T)$ establish $\mathrm{CeCrGe}_{3}$ as a moderate heavy fermion compound with a quasiparticle mass renormalization factor of $\sim 45$.
\end{abstract}

PACS numbers: 75.30.Mb, 75.30.-m, 75.40.-s, 72.15.Jf

\section{INTRODUCTION}

Superconductivity in iron pnictide was discovered at the boundary of magnetically ordered and non-ordered state of Fe-magnetism. The spin-density-wave type antiferromagnetic order in $\mathrm{RFeAsO}(\mathrm{R}=\mathrm{La}, \mathrm{Ce}, \mathrm{Pr}, \mathrm{Nd}, \mathrm{Sm})$ and $\mathrm{AFe}_{2} \mathrm{As}_{2}(\mathrm{~A}=\mathrm{Ca}, \mathrm{Ba}, \mathrm{Sr}, \mathrm{Eu})$ could be suppressed by carrier doping ${ }^{1}-\underline{\underline{3}}$ or by application of chemical/externally applied pressure $\stackrel{\underline{4}-\underline{6}}{ }$ One may expect to find similar interesting results by tuning the magnetic state of $\mathrm{Cr}$ moments as well. Recently Cr-magnetic ordering at relatively low temperature has been reported in $\mathrm{RCrGe}_{3}$ system where $\mathrm{R}$ stands for rare-earth elements. $\stackrel{7}{\mathrm{C}}$ On the other hand, Ce-based ternary germanide, $\mathrm{RTGe}_{3}(\mathrm{R}=$ rare-earth elements and $\mathrm{T}=3 d, 4 d, 5 d$ elements) system exhibits some interesting magnetic properties. For instance, $\mathrm{CeRhGe}_{3}$ shows a weak Kondo effect along with three magnetic phase transitions $\underline{26} \mathrm{Ce}$ moments order antiferromagnetically below $T_{N}=5.5 \mathrm{~K}$ in $\mathrm{CeNiGe}_{3}[9]$ which exhibits pressure induced superconductivity $\underline{10}$ below $0.48 \mathrm{~K}$ in a wide pressure range from 4 to $10 \mathrm{GPa}$. Furthermore, a complex magnetic phase diagram is found in $\mathrm{CeCoGe}_{3}$ [11] which also exhibit superconductivity under the application of external pressure. ${ }^{12}$

The study of the anomalous physical properties close to the borderline between magnetically ordered and nonmagnetic ground states is currently of intense interest in the contemporary condensed matter physics research. This point of instability achieved by non-thermal tuning parameters between two stable phases of matter is called Quantum Critical Point (QCP). Interestingly, a quantum critical behavior is evidenced in doped $\mathrm{CeCoGe}_{3}[13,14]$. The situation may, however, get more complicated in $\mathrm{CeCrGe}_{3}$ due to the presence of Cr-magnetic ordering. Very recently the suppression of $\mathrm{Cr}$ ferromagnetic ordering has been reported in $\operatorname{LaV}_{x} \mathrm{Cr}_{1-x} \mathrm{Ge}_{3}$ system 15 with a possibility for the presence of a QCP in this system. Such possibility also exists in the corresponding Ce-system and this motivates us to study $\mathrm{CeCrGe}_{3}$ in detail.

The compounds $\mathrm{RCrGe}_{3}$ are reported to crystallize in the hexagonal perovskite structure (space group $\left.P 6_{3} / m m c\right)^{?} \stackrel{7}{=} \mathrm{A}$ preliminary investigation based on magnetization measurements of $\mathrm{CeCrGe}_{3}$ reports ferromagnetic ordering of $\mathrm{Cr}$-moments below $66 \mathrm{~K}$; much less is known about the Ce-magnetism and its interaction with Cr-moments in this compound. We herein report comprehensive study of low temperature properties of $\mathrm{CeCrGe}_{3}$ by means of x-ray absorption spectroscopy (XAS), magnetic susceptibility $\chi(T)$, isothermal magnetization $\mathrm{M}(\mathrm{H})$, electrical resistivity $\rho(T)$, specific heat $\mathrm{C}(T)$ and thermoelectric power $\mathrm{S}(T)$ measurements. In order to explore the role of $4 f$-electron we have also measured the properties of the corresponding $4 f^{0}$-electron analog $\mathrm{LaCrGe}_{3}$. Our results corroborate that $\mathrm{Cr}$ ions order ferromagnetically below $70 \mathrm{~K}$ in $\mathrm{CeCrGe}_{3}$ and Ceions possess a stable $3+$ valance state in this compound. Our observation of a large value of Sommerfeld coefficient $\gamma$, obtained from the specific heat measurement, even in the magnetically ordered state is quite remarkable in this context. The Kondo lattice type of the resistivity and the large $\gamma$ tempt us to believe that $\mathrm{CeCrGe}_{3}$ is a moderate heavy fermion system.

\section{EXPERIMENTAL DETAILS}

Polycrystalline samples of $\mathrm{LaCrGe}_{3}$ and $\mathrm{CeCrGe}_{3}$ were prepared by arc melting the high purity elements, La and Ce pieces (99.9\%, Alfa-Aesar), Cr and Ge chips (99.99\% Sigma Aldrich), taken in stoichiometric ratio on water cooled copper hearth under an argon atmosphere. The samples were flipped after each melting and were melted several times to ensure homogeneity. In order to homogenize and remove the impurity phases the melted but- 
tons were wrapped in Ta foil and annealed in an evacuated quartz tube at $900^{\circ} \mathrm{C}$ for one week. The phase purities of the annealed samples were checked by powder x-ray diffraction (XRD) using $\mathrm{Cu}-K_{\alpha}$ radiation and metallographic examinations. The absorption spectra of $\mathrm{CeCrGe}_{3}$ at the $\mathrm{Ce}-L_{I I I}$ edge $(\mathrm{E}=5723 \mathrm{eV})$ have been recorded in transmission mode at the EXAFS beam line A1 of the Hamburg synchrotron radiation facility (HASY-LAB at DESY) using the Si (111) double crystal monochromator. In order to perform the XAS study finely grounded/powdered sample of $\sim 12 \mathrm{mg}$ with particle size smaller than $20 \mu \mathrm{m}$ was mixed with $\mathrm{B}_{4} \mathrm{C}$ and $\mathrm{PE}$ powder and pelletized to a $10 \mathrm{~mm}$ disc. The absorption behavior has been determined in the energy range from $5500 \mathrm{eV}$ up to the $\mathrm{Cr} \mathrm{K}$ absorption edge $(5989 \mathrm{eV})$ with a minimal step size of $\Delta \mathrm{E}=0.25 \mathrm{eV}$ near the Ce$L_{I I I}$ edge $(5723 \mathrm{eV})$. The pellet was mounted in an $\mathrm{Ox}-$ ford gas flow cryostat to realize temperatures down to the boiling point of liquid helium. The spectra showed an absorption edge step of $\Delta \mu=0.8$ which represents the difference of the low and high energy background functions at the absorption edge. The $\left(\mathrm{Ce}^{3+}\right)$-reference $\mathrm{CePO}_{4}$ was measured simultaneously at ambient temperature and was used for energy calibration. Evaluation of spectra, energy calibration and normalization have been performed with the Athena module of the Horae software package ${ }^{16}$ Magnetic measurements were carried out using a commercial Superconducting Quantum Interference Device (SQUID) magnetometer (MPMS Quantum Design). Electrical resistivity measurements were carried out in the temperature range 2-300 K using ac transport option of physical property measurement system (PPMS, Quantum Design). The specific heat was measured by relaxation method in PPMS. The thermoelectric power (TEP) was measured using thermal transport option of PPMS.

\section{RESULTS AND DISCUSSION}

The crushed polycrystalline samples were characterized by x-ray diffraction with $\mathrm{Cu}-K_{\alpha}$ radiation to determine the single phase nature and the crystal structure. Powder x-ray data were analyzed by Rietveld refinement using FullProf software $\stackrel{17}{ }$ The refinement reveals the single phase nature of the samples. The lattice parameters for $\mathrm{CeCrGe}_{3}$ and $\mathrm{LaCrGe}_{3}$, given in Table I, are in good agreement with those reported in the literature ${ }^{7}$ The room temperature XRD pattern and Rietveld refinement profile are shown in Fig 1. Compositional homogeneity was checked by performing metallographic examinations like Scanning electron microscopy (SEM) and Energy dispersive $\mathrm{x}$-ray spectroscopy.

To elucidate the valence state of $\mathrm{Ce}$ in $\mathrm{CeCrGe}_{3}$ we performed Ce- $L_{I I I}$ edge x-ray absorption study. The spectra of $\mathrm{CeCrGe}_{3}$ and the $\left(\mathrm{Ce}^{3+}\right)$ - reference compound $\mathrm{CePO}_{4}$ show one single white line at the Ce- $L_{I I I}$ edge with a typical broadening in the spectra of an intermetallic com-

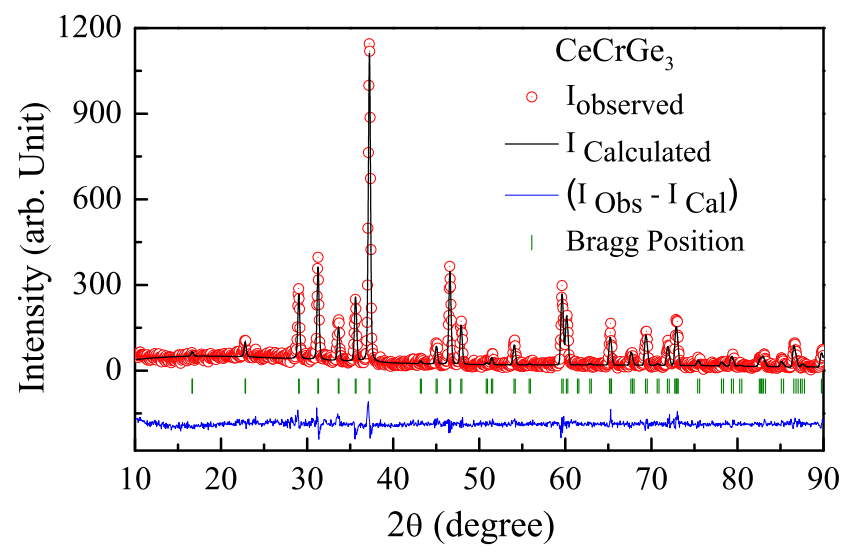

FIG. 1: (Color online) The powder x-ray diffraction pattern of $\mathrm{CeCrGe}_{3}$ recorded at room temperature. The solid line through the experimental points is the Rietveld refinement profile calculated for the $\mathrm{BaNiO}_{3}$-type hexagonal perovskite structure (space group $\mathrm{P}_{3} / \mathrm{mmc}$ ). The short vertical bars mark the Bragg peak positions. The lowermost curve represents the difference between the experimental and calculated intensities.

TABLE I: Table I: Crystallographic data for $\mathrm{RCrGe}_{3}(\mathrm{R}=$ La,Ce) obtained from the structural Rietveld refinement of powder XRD data.

\begin{tabular}{ccc}
\hline \hline Structure & $\mathrm{BaNiO}_{3}$-type Hexagonal \\
Space group & $P 6_{3} / m m c$ & \\
& & \\
Lattice parameters & $\mathrm{LaCrGe}_{3}$ & $\mathrm{CeCrGe}_{3}$ \\
$a(\AA)$ & 6.198 & 6.150 \\
$c(\AA)$ & 5.765 & 5.719 \\
$V_{\text {cell }}\left(\AA^{3}\right)$ & 191.79 & 187.32 \\
\hline \hline
\end{tabular}

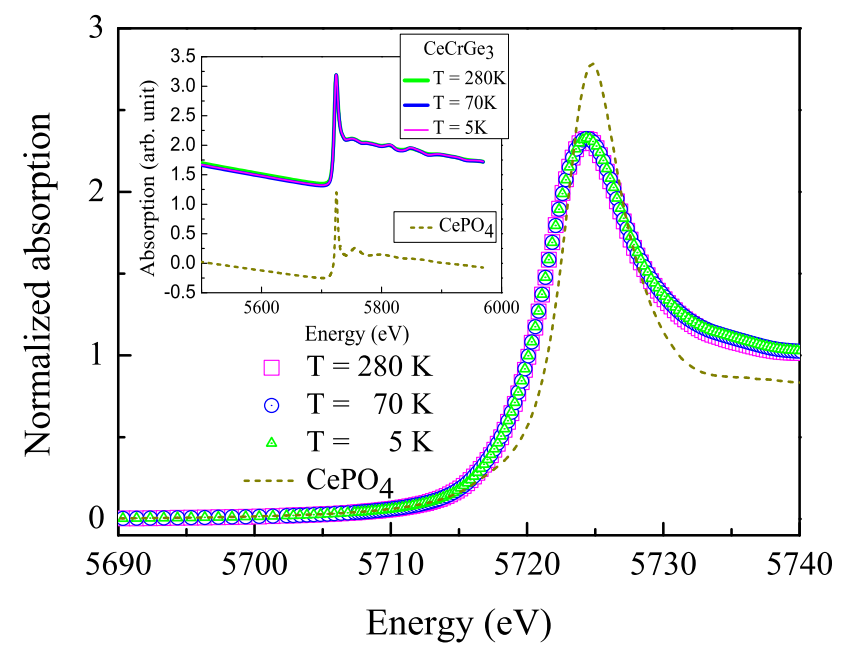

FIG. 2: (Color online) Ce- $L_{I I I}$ edge x-ray absorption of $\mathrm{CeCrGe}_{3}$ at $280 \mathrm{~K}, 70 \mathrm{~K}$ and $5 \mathrm{~K}$ in comparison to the $(\mathrm{Ce} 3+)$ reference spectrum of $\mathrm{CePO}_{4}$. (Inset: un-normalised spectra). 
pound. Neither the spectra of $\mathrm{CeCrGe}_{3}$ nor its derivative indicate an additional $\left(\mathrm{Ce}^{4+}\right)$ contribution which would lead to an additional absorption line at approximately $5730 \mathrm{eV}$. The spectrum is temperature independent down to $\mathrm{T}=5 \mathrm{~K}$ and consequently the $\left(\mathrm{Ce}^{3+}\right)$ valence state remains unchanged in the examined range $5 \mathrm{~K}<\mathrm{T}<$ $280 \mathrm{~K}$.

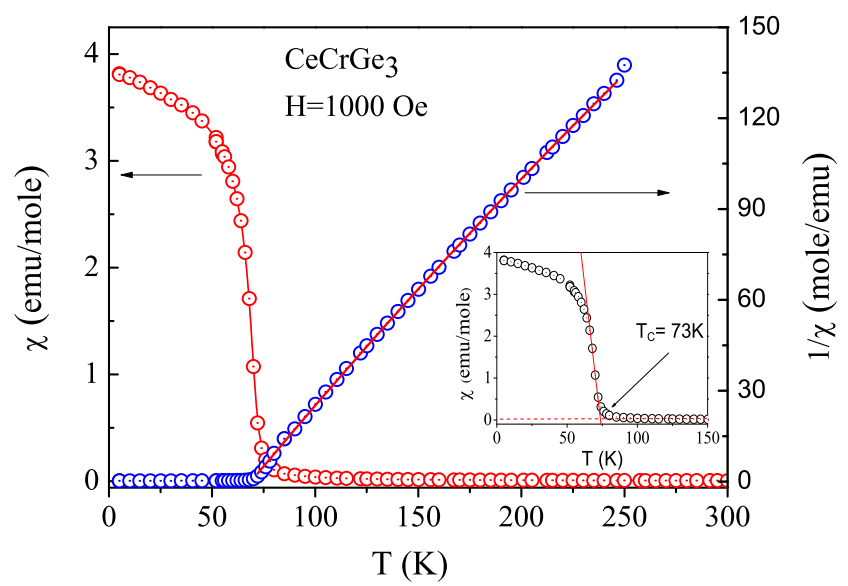

FIG. 3: (Color online) Field-cooled (FC) dc magnetic susceptibility and inverse susceptibility of $\mathrm{CeCrGe}_{3}$. Inset: Magnetic susceptibility in the temperature range $2 \mathrm{~K}-150 \mathrm{~K}$. The Magnetic ordering temperature is represented by the meeting point of the solid and dotted lines.

The temperature dependence of the magnetic susceptibility $\chi(T)$ of $\mathrm{CeCrGe}_{3}$ is shown in Fig. 3 in the temperature range $2-300 \mathrm{~K}$ under an applied field of $0.1 \mathrm{~T}$. At high temperature $\chi(T)$ follows the modified Curie-Weiss behavior, $\chi(T)=\chi_{0}+C /\left(T-\theta_{P}\right)$ where $\chi_{0}$ is temperature independent susceptibility, $\mathrm{C}$ is the Curie constant and $\theta_{P}$ is the Weiss temperature. Fig. 3 also displays the inverse magnetic susceptibility as a function of temperature along with the fitting of $\chi^{-1}(\mathrm{~T})$ data with the modified Curie-Weiss behavior in the high temperature range. The effective moment $\mu_{\text {eff }}$ values calculated from the Curie constants are found to be $2.66 \mu_{B}$ and $3.18 \mu_{B}$ for $\mathrm{LaCrGe}_{3}$ and $\mathrm{CeCrGe}_{3}$ respectively. The observation of this increased effective moment value for $\mathrm{CeCrGe}_{3}$ is consistent with the trivalent state of Ce-ions revealed by XAS measurement. It is to be noted in this context that these values are higher than those reported in Ref. 7 . For LaCrGe3 a value of $2.5 \mu_{B}$ has been reported recently by Lin et al. [15] which is nearly same as the value obtained by us. For $\mathrm{CeCrGe}_{3} \mu_{\text {eff }}$ is higher than the calculated value for free $\mathrm{Ce}^{3+}: 2.54 \mu_{B}$. As it is confirmed by XAS study that $\mathrm{Ce}$ in $\mathrm{CeCrGe}_{3}$ possesses a stable $3+$ valance state with a theoretical value of $\mu_{e f f}=2.54 \mu_{B}$, Ce contribution to the Curie constant $(C)$ is $C_{C e}=0.81$ emu K/mol. So, now by subtracting $C_{C e}$ from $C(=1.26$ emu $\mathrm{K} / \mathrm{mol}$ ) we can determine the $\mathrm{Cr}$ contribution to the Curie constant, $C_{C r}$, which gives an effective moment value of $\mu_{\text {eff }}(\mathrm{Cr})=1.81 \mu_{B}$ [see Ref. No 18 for analysis]. This $\mu_{\text {eff }}(\mathrm{Cr})$ value is less than that for $\mathrm{Cr}^{4+}\left(2.8 \mu_{B}\right)$ or $\mathrm{Cr}^{3+}\left(3.8 \mu_{B}\right)$. The reduced $\mathrm{Cr}$ moment suggests an itinerant character of $\mathrm{Cr}$ magnetism. The positive value of Weiss temperature $\theta_{P}$ suggests the presence of dominant ferromagnetic interactions in the system. The Curie temperatures $T_{C}$ were derived by extrapolating the maximum slope $(\mathrm{d} \chi / \mathrm{d} T)$ to zero as shown in the inset of Fig. 3. The effective moments $\mu_{\text {eff }}$, Weiss temperatures $\theta_{P}$ and Curie temperatures $T_{C}$ are summarized in Table II.

Fig. 4 represents the magnetic isotherms $\mathrm{M}(\mathrm{H})$ of $\mathrm{CeCrGe}_{3}$ measured at $1.8 \mathrm{~K}$ and $300 \mathrm{~K}$ under applied magnetic fields up to $7 \mathrm{~T}$. In the ordered state $\mathrm{M}(\mathrm{H})$ at $1.8 \mathrm{~K}$ shows a rapid increase up to a value of $\sim 0.6 \mu_{B}$ at low field followed by slow increase beyond $0.2 \mathrm{~T}$. This may be due to possible canted ferromagnetic ordering of $\mathrm{Cr}-$ moments as revealed in $\mathrm{LaCrGe}_{3}$ by neutron diffraction study $\frac{19}{19}$ At $1.8 \mathrm{~K}$ magnetization reaches a value of $0.8 \mu_{B}$ /f.u. at $7 \mathrm{~T}$ for $\mathrm{CeCrGe}_{3}$ which is lower than the value $1.3 \mu_{B} /$ f.u. at $7 \mathrm{~T}$ obtained for $\mathrm{LaCrGe}_{3}$. The reduced value for $\mathrm{CeCrGe}_{3}$ could arise due to crystal field effect/Kondo effect/ interaction between the Ce and $\mathrm{Cr}$ sublattice. At $300 \mathrm{~K} \mathrm{M}(\mathrm{H})$ is almost linear in $\mathrm{H}$ as expected in the paramagnetic state.

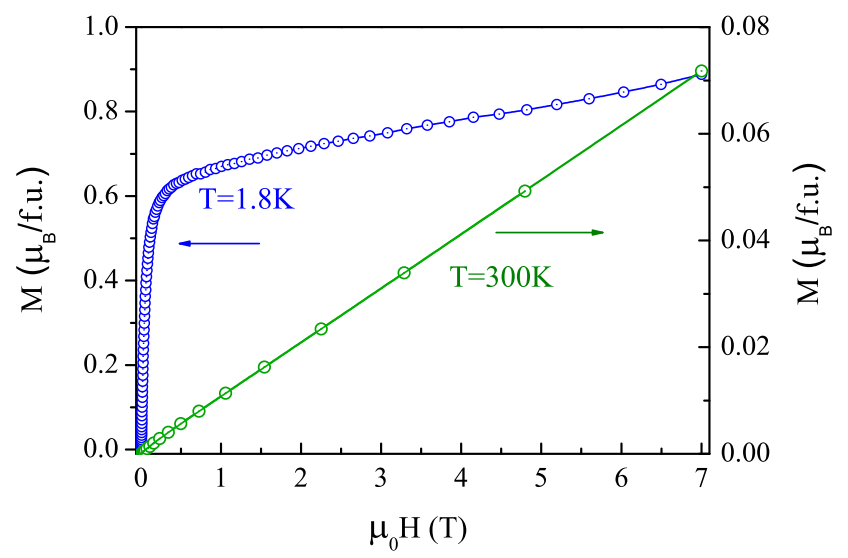

FIG. 4: (Color online) Magnetic field dependence of isothermal magnetization data, $\mathrm{M}(\mathrm{H})$ of $\mathrm{CeCrGe}_{3}$ measured at constant temperatures $1.8 \mathrm{~K}$ and $300 \mathrm{~K}$.

The electrical resistivity $\rho(T)$ of $\mathrm{LaCrGe}_{3}$ and $\mathrm{CeCrGe}_{3}$ measured in the temperature range $2-300 \mathrm{~K}$ is shown in Fig. 5(a). While $\mathrm{LaCrGe}_{3}$ exhibits a typical metallic behavior with residual resistivity $\rho_{0} \sim$ $65 \mu \Omega \mathrm{cm}$ at $2 \mathrm{~K}$ and residual resistivity ratio $\mathrm{RRR}$ $=\rho_{300 \mathrm{~K}} / \rho_{2 \mathrm{~K}} \sim 4.3$, the electrical resistivity profile of $\mathrm{CeCrGe}_{3}$ interestingly exhibits Kondo like behavior. For $\mathrm{CeCrGe}_{3}$, the resistivity increases with decreasing temperature down to $70 \mathrm{~K}$ followed by a drastic fall of electrical resistivity below $70 \mathrm{~K}$ as a consequence of reduction of spin disorder resistivity due to Cr-moment ordering. Below $30 \mathrm{~K}$ the resistivity again increases similar to the case of Kondo insulator/semiconductor. Here, the temperature at which the resistivity anomaly occurs has been denoted by $T_{c}^{(\rho-T)}$ and is summarized in Table II. The magnetic contribution to the resistivity $\rho_{\text {mag }}$ obtained 
by subtracting the resistivity of $\mathrm{LaCrGe}_{3}$ from that of $\mathrm{CeCrGe}_{3}$ is depicted in Fig. 5(b). The temperature variation of $\rho_{\text {mag }}$ follows - $\ln T$ behavior in two different temperature regions which we believe is associated with the presence of Kondo effect as seen in many Ce-based Kondo Lattice/Heavy fermion systems. These two regions are separated by the FM transition around $70 \mathrm{~K}$. A sharp increase of $\rho_{\text {mag }}(\mathrm{Ce}-\mathrm{La})$ below $86 \mathrm{~K}$ is an artificial effect of the subtraction process because $T_{C}=88 \mathrm{~K}$ of the Lasystem is larger than $T_{C}=68 \mathrm{~K}$ of the Ce-system. We
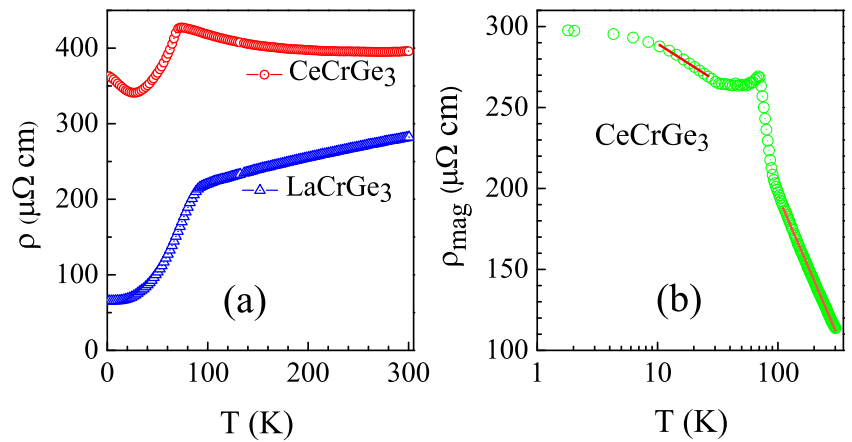

FIG. 5: (Color online) (a) Electrical resistivity of $\mathrm{LaCrGe}_{3}$ and $\mathrm{CeCrGe}_{3}$, (b) Magnetic contribution to the electrical resistivity. Solid lines are fit to the equation $\rho_{\text {mag }}=\rho_{0}-\mathrm{C}_{k}$ $\ln T$

have analyzed the data of $\rho_{m a g}$ using the expression:

$$
\rho_{m a g}=\rho_{0}-C_{k} \ln T
$$

where $\rho_{0}$ is the spin disorder resistivity and $\mathrm{C}_{k}$ is the Kondo coefficient. The fitting to this equation at two different temperature regions are displayed by the solid lines in Fig 5(b). The low temperature (10 K - $25 \mathrm{~K})$ - $\ln T$ behaviour represents the Kondo scattering in the crystal field ground state whereas $-\ln T$ dependence in the high temperature $(110 \mathrm{~K}-300 \mathrm{~K})$ part is due to Kondo effect in the excited multiplets. ${ }^{20}$ The high temperature $\ln T$ dependence is one of the characteristic features of the dense Kondo systems.

Sometimes Kondo system can have large value of Sommerfeld coefficient $\gamma$. In order to get the information about the linear term $\gamma$ in $\mathrm{CeCrGe}_{3}$ system exhibiting Kondo like behavior, we have performed specific heat measurements. Fig. 6(a) represents the temperature dependence of specific heat $\mathrm{C}(T)$ of $\mathrm{RCrGe}_{3}$, for $\mathrm{R}=\mathrm{La}$ and Ce. The anomalies at $\sim 84 \mathrm{~K}$ and $67 \mathrm{~K}$ as seen in $\mathrm{C}(T)$ data for $\mathrm{LaCrGe}_{3}$ and $\mathrm{CeCrGe}_{3}$ respectively are consistent with the anomalies observed in temperature dependence of magnetic susceptibility as well as in electrical resistivity. The bulk nature of ferromagnetic ordering as evidenced by magnetic measurements and reported in the literature $e^{7.15}$ is confirmed by the anomaly in $\mathrm{C}(T)$ data at $84 \mathrm{~K}$ and $67 \mathrm{~K}$ for $\mathrm{LaCrGe}_{3}$ and $\mathrm{CeCrGe}_{3}$ respectively. The low temperature specific heat data below $20 \mathrm{~K}$ can be fitted with the equation, (a)


FIG. 6: (Color online) (a) Specific heat of $\mathrm{RCrGe}_{3}$ as a function of $T$. Inset shows the low temperature fit to the equation $C(T)=\gamma T+\beta T^{3}$ for $\mathrm{CeCrGe}_{3}$. (b) $\mathrm{C}_{4 f}$ and magnetic entropy $\left(\mathrm{S}_{4 f}\right)$ as a function of temperature. Inset: $\mathrm{C}_{4 f}$ in the temperature range $0.4 \mathrm{~K}$ to $1.8 \mathrm{~K}$.

$$
C(T)=\gamma T+\beta T^{3}
$$

where $\gamma T$ is the electronic contribution to the specific heat, $\beta T^{3}$ is the phononic contribution to the specific heat [inset of the Fig. 6(a)]. The value of Debye temperature $\Theta_{D}$ can be estimated from $\beta$ using the relation, ${ }^{21}$

$$
\Theta_{D}=\left(\frac{12 \pi^{4} n R}{5 \beta}\right)^{1 / 3}
$$

where $\mathrm{R}$ is the molar gas constant and $\mathrm{n}=5$ is the number of atoms per formula unit (f.u.). We have listed the obtained values of $\gamma$ and $\Theta_{D}$ for the two compounds in Table II. The Sommerfeld coefficient $(\gamma)$ for $\mathrm{CeCrGe}_{3}$ is found out to be $130 \mathrm{~mJ} / \mathrm{mol} \mathrm{K}^{2}$. This value is very large compared to the $\gamma$ value $2.9 \mathrm{~mJ} / \mathrm{mol} \mathrm{K}^{2}$ obtained for $\mathrm{LaCrGe}_{3}$ having $4 f^{0}$ configuration. This $\gamma$ value of 
TABLE II: Summary of magnetization, electrical resistivity and specific heat data.

\begin{tabular}{ccccccc}
\hline \hline Sample & $\mu_{e f f}\left(\mu_{B}\right)$ & $\theta_{P}(\mathrm{~K})$ & $T_{c}^{(m a g)}(\mathrm{K})$ & $T_{c}^{(\rho-T)}(\mathrm{K})$ & $\Theta_{D}(\mathrm{~K})$ & $\gamma\left(\mathrm{mJ} / \mathrm{mol} \mathrm{K}^{2}\right)$ \\
\hline $\mathrm{LaCrGe}_{3}$ & 2.66 & 87 & 88 & 88 & 253 & 2.89 \\
$\mathrm{CeCrGe}_{3}$ & 3.18 & 67 & 73 & 68 & 250 & 130 \\
\hline \hline
\end{tabular}

$\mathrm{CeCrGe}_{3}$ is comparable with that found in case of moderate heavy fermion system like $\mathrm{CeFeGe}_{3}[21], \mathrm{Ce}_{2} \mathrm{Rh}_{3} \mathrm{Ge}_{5}$ [22], $\mathrm{CeCoGe}_{3}$ [11], $\mathrm{YbNi}_{2} \mathrm{~B}_{2} \mathrm{C}$ [23] etc. Assuming that the quasi-particle mass renormalization in $\mathrm{LaCrGe}_{3}$ is negligible, the renormalization factor for quasi-particle density of states due to $4 \mathrm{f}$ correlations in $\mathrm{CeCrGe}_{3}$ can be obtained by the ratio $\gamma\left(\mathrm{CeCrGe}_{3}\right) / \gamma\left(\mathrm{LaCrGe}_{3}\right)$ which comes out to be $\sim 45$. Hence, the quasi-particle mass in $\mathrm{CeCrGe}_{3}$ can be roughly estimated as $\mathrm{m}^{*} \approx 45 \mathrm{~m}_{e}$, where $\mathrm{m}_{e}$ represents the free electron mass. Although a band structure calculation or de Haas-van Alphen studies on single crystal of $\mathrm{CeCrGe}_{3}$ is required for a better estimate of $\mathrm{m}^{*}$, the effective mass estimated in this way was found to be very close to the experimentally observed value for $\mathrm{RRhSi}_{3}$ system $(\mathrm{R}=\mathrm{La}$ and $\mathrm{Ce})$ where $\mathrm{LaRhSi}_{3}$ has $\gamma=6 \mathrm{~mJ} / \mathrm{mol} \mathrm{K}^{2}[25]$ and $\mathrm{CeRhSi}_{3}$ has $\gamma$ $=120 \mathrm{~mJ} / \mathrm{mol} \mathrm{K}^{2}[26]$. Thus the ratio of $\gamma$ gives $\mathrm{m}^{*}=$ $20 \mathrm{~m}_{e}$ which is very close to the experimentally observed $\mathrm{m}^{*}=19 \mathrm{~m}_{e}$ to $24 \mathrm{~m}_{e}$ in de Haas-van Alphen studies on $\mathrm{CeRhSi}_{3}[27,28]$. Thus, the observation of a typical Kondo lattice behavior in electrical resistivity and an enhanced $\gamma$ value suggest that $\mathrm{CeCrGe}_{3}$ can be classified as a member of the heavy fermion system. The observation of heavy fermion behavior even in the presence of ferromagnetic order having large ordering temperature is remarkable in this system.

In order to obtain the Ce- $4 f$ contribution to the specific heat $\left(\mathrm{C}_{4 f}\right)$, we have subtracted the heat capacity of $\mathrm{LaCrGe}_{3}$ from that of $\mathrm{CeCrGe}_{3}$ which is displayed in a plot of $\mathrm{C}_{4 f} / T$ versus $T$, Fig $6(\mathrm{~b})$. The first observation is that the $T$ dependence of the $\mathrm{C}_{4 f} / T$ value is very flat. Besides the small sharp anomaly at $T=2.8 \mathrm{~K}$ there is only a weak and broad maximum at $15 \mathrm{~K}$ followed by a smooth decrease to higher $T$, which however amount to less than $30 \%$ between $15 \mathrm{~K}$ and $50 \mathrm{~K}$. The overall $\mathrm{C}_{4 f} / T$ value is enhanced, of the order of $120 \mathrm{~mJ} / \mathrm{mol} \mathrm{K}^{2}$. For a stable trivalent $\mathrm{Ce}^{3+}$ without Kondo interaction, one would expect a polarization of the Ce-moment in the exchange field produced by $\mathrm{FM}$ ordering of $\mathrm{Cr}$, possibly followed by ordering of Ce on its own at lower temperatures. This would result in a clear Schottky anomaly due to Zeeman splitting, possibly followed by a sharp anomaly, and ending in a pronounced decrease to lowest $\mathrm{T}$ since the Ce system is then in a fully polarized state $\stackrel{30,31}{ }$ This scenario does not fit at all to the experimental observations made here, namely, $\mathrm{C}_{4 f} / T$ remains large up to the lowest $T$ investigated, $0.4 \mathrm{~K}$ (inset Fig. 6(b)), much more similar to the expected behavior of the heavy fermion system. This large $\mathrm{C}_{4 f} / T$ value at lowest $T$ implies strong fluctuations extending down to $T=$ $0 \mathrm{~K}$. Assuming these fluctuations are due to Kondo effect,
$\mathrm{C}_{4 f} / T$ allows for an estimate of the Kondo temperature $T_{K}$ using the theoretical expression derived by Rajan et $\mathrm{al}^{29,32,33}$

$$
\left(\frac{C_{4 f}}{T}\right)_{T=0}=\frac{(\nu-1) \pi R}{6 T_{0}}
$$

where $\mathrm{R}$ is the gas constant, $\nu$ is the degeneracy of the lowest level concerned and $T_{0}$ is a scaling temperature which is related to the Kondo temperature $T_{K}$ by $T_{K} / T_{0}$ $=1.290$. Taking ground state degeneracy $\nu=2$, and $\left(\mathrm{C}_{4 f} / T\right)_{T=0}=0.117 \mathrm{~J} / \mathrm{mol} \mathrm{K}^{2}, T_{K}=48 \mathrm{~K}$ and taking $\nu$ $=4$ we get $T_{K}=143 \mathrm{~K}$.

Furthermore, the $4 f$-entropy obtained from the integration of $\mathrm{C}_{4 f} / T$ increases continuously at least up to $50 \mathrm{~K}$ where it has reached a value close to Rln2 [Fig $6(\mathrm{~b})]$. Even above $50 \mathrm{~K}$ the magnetic entropy $\mathrm{S}_{4 f}(T)$ continues to increase with $\mathrm{T}$, this implies that the energy splitting to first excited CEF level should be smaller than $150 \mathrm{~K}$. However, the absence of saturation behavior towards Rln2 implies that this splitting is not well defined (resolved). The high $T_{K}$ obtained from the Sommerfeld coefficient provides a first simple explanation, since a strong correlation that is responsible for high gamma value would lead to a broadening of the CEF level of the same order of magnitude as the splitting. However, the exchange field due to the FM ordering of $\mathrm{Cr}$ moments provides a further mechanism, but the exchange field needed is huge, since it has to lead to Zeeman splitting of the order of $150 \mathrm{~K}$. Inelastic neutron scattering experiments are required to have a clear idea about the CEF level scheme in this system.

As a complementary probe for characteristics of the Kondo effect, we performed thermopower measurements. Fig.7 shows the thermopower $\mathrm{S}(T)$ of $\mathrm{LaCrGe}_{3}$ and $\mathrm{CeCrGe}_{3}$ between 5 and $300 \mathrm{~K}$. Additionally we calculated the magnetic contribution $\mathrm{S}_{\text {mag }}(T)$ to the thermopower of $\mathrm{CeCrGe}_{3}$ by the Gorter-Nordheim relation,

$$
S * \rho=S_{m a g} * \rho_{\text {mag }}+S_{0} * \rho_{0}
$$

where $\mathrm{S}_{0}$ and $\rho_{0}$ are the data for the isostructural La compound. Typically, Ce-based Kondo systems exhibit (a) enhanced thermopower values, which are 10-100 times larger than that of simple metals like $\mathrm{Cu}, \stackrel{34}{34}$ and (b) a positive sign of $\mathrm{S}(T) \stackrel{35}{\underline{5}}$ For well-defined CEF resonances and a low $T_{K}$ one also finds (c) two maxima: one around the Kondo temperature $T_{K}$ and the second around a temperature corresponding to roughly 0.3 to 0.6 of the $\mathrm{CEF}$ splitting, $\underline{34}$ They reflect Kondo scattering on the ground state and on the excited CEF states, respectively. Our data show (a) indeed a very high value of both $\mathrm{S}_{\text {mag }}(T)$ 


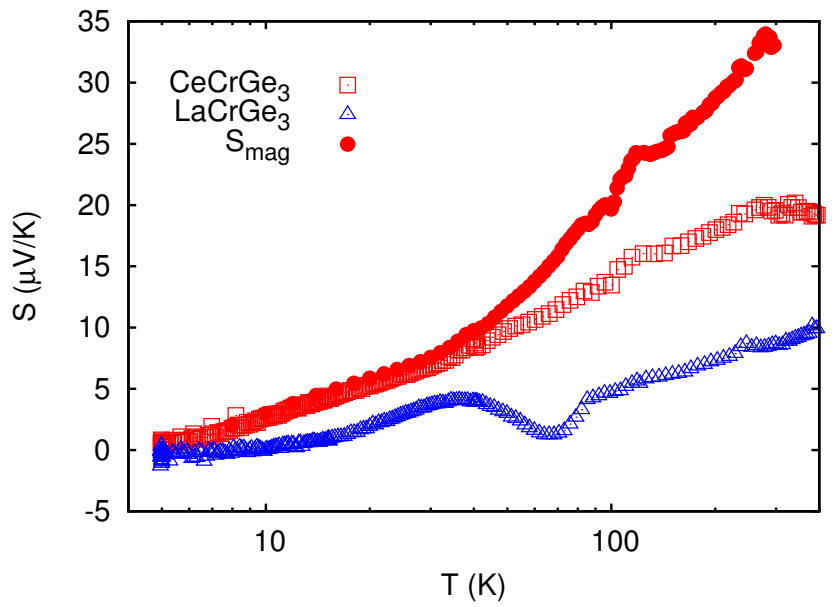

FIG. 7: (Color online) Thermoelectric power of $\mathrm{LaCrGe}_{3}$ and $\mathrm{CeCrGe}_{3}$ as a function of temperature.

$(35 \mu \mathrm{V} / \mathrm{K})$ and $\mathrm{S}(T)(20 \mu \mathrm{V} / \mathrm{K})$ of $\mathrm{CeCrGe}_{3}$. Additionally we find (b) that $\mathrm{S}(T)$ is positive in the whole temperature range. Both properties indicate that $\mathrm{CeCrGe}_{3}$ is a Kondo system. Concerning (c) we, however, do not see any extrema in $\mathrm{S}_{m a g}(T)$, but a monotonic increase up to room temperature. Since we expect a relatively high Kondo temperature from our specific heat measurements and additionally do not find clear signatures of the CEF levels in the specific heat either, we are far from the conditions mentioned for (c). Indeed, it is known from theoretical calculations describing experiments e.g. on $\mathrm{CeRu}_{2} \mathrm{Ge}_{2}$, that the temperature dependence of the thermopower can vary drastically and can even become monotonic depending on the Kondo scale and the CEF splitting ${ }^{34}$ In the $\mathrm{LaCrGe}_{3}$ system we additionally find a broad hump between 30 and $60 \mathrm{~K}$, which is believed to be related to the ordering of the $\mathrm{Cr}$ moments.

\section{CONCLUSIONS}

We have provided experimental evidence for the presence of Kondo effect/heavy fermion behavior of the trivalent $\mathrm{Ce}$ compound $\mathrm{CeCrGe}_{3}$ in presence of the ferromagnetic order due to itinerant Cr-moments. While the conclusive evidence of the trivalent nature was obtained from XAS, the presence of Kondo behavior was revealed by electrical resistivity, thermoelectric power and heat capacity data. The heavy fermion nature was revealed using the heat capacity measurement. Specific heat data show an enhanced $\gamma\left(130 \mathrm{~mJ} / \mathrm{mol} \mathrm{K}^{2}\right)$ value for $\mathrm{CeCrGe}_{3}$ compared to $2.9 \mathrm{~mJ} / \mathrm{mol} \mathrm{K}^{2}$, obtained for $\mathrm{LaCrGe}_{3}$. Although magnetic order of $\mathrm{Cr}$ was known from earlier magnetization data, our heat capacity data confirms the bulk nature of the magnetic order. Hence, the compound $\mathrm{CeCrGe}_{3}$, having a high $T_{K}(\sim 100 \mathrm{~K})$ and a trivalent integral Ce valence state at room temperature as well as at low temperature, appears to be a rare example of $\mathrm{Ce}$ based system exhibiting heavy fermion behavior even in the magnetically ordered state.

\section{ACKNOWLEDGEMENTS}

We would like to thank Dr. V. K. Anand for useful discussion. This work is partially supported by Council of Scientific and Industrial Research (CSIR), New Delhi (Grant No. 80(0080)/12/ EMR-II).
* Electronic address: zakir@iitk.ac.in

${ }^{1}$ Hiroki Takahashi, Kazumi Igawa, KazunobuArii, Yoichi Kamihara, Masahiro Hirano and Hideo Hosono; Nature, 453, 376 (2008).

2 Athena S. Sefat, Rongying Jin, Michael A. McGuire, Brian C. Sales, David J. Singh, and David Mandrus; Phys. Rev. Lett. 101, 117004 (2008).

3 Anupam, P L Paulose, H S Jeevan, C Geibeland Z Hossain; J. Phys.: Condens. Matter 21265701 (2009).

4 Marianne Rotter, Marcus Tegel and Dirk Johrendt; Nature Materials 101, 107006 (2008).

5 Tuson Park, Eunsung Park, Hanoh Lee, T Klimczuk, E D Bauer, F Ronning and J D Thompson; J. Phys.: Condens. Matter 20 322204, (2008).

6 Zhi Ren, Qian Tao, Shuai Jiang, ChunmuFeng, Cao Wang, Jianhui Dai, Guanghan Cao, and Zhu'anXu. Phys. Rev. Lett. 102, 137002 (2009).

7 Haiying Bie,Oksana Ya. Zelinska, Andriy V. Tkachuk and Arthur Mar ; Chem. Mater 194613 (2007).

8 Yuji Muro, DuhwaEom, Naoya Takeda and Masayasu Ishikawa. J. Phys. Soc. Jpn. 673601 (1998).

9 P. Manfrinetti, S.K. Dhar, R. Kulkarni, A.V. Morozkin,
Solid State Communications 135, 444 (2005).

10 M Nakashima, K Tabata, A Thamizhavel, T C Kobayashi, M Hedo, Y Uwatoko, K Shimizu, R Settai and YÖnuki, J.Phys.: Condens. Matter 16 L255 (2004).

11 V. K. Pecharsky, O.-B. Hyun, and K. A. Gschneidner, Jr.; Phys. Rev. B. 4711839 (1993).

12 R. Settai, I. Sugitani, Y. Okuda, A. Thamizhavel, M. Nakashima, Y. Ōnuki, H. Harima; Journal of Magnetism and Magnetic Materials 310844 (2007).

13 Duhwa Eom, Masayasu Ishikawa, Jiro Kitagawa and Naoya Takeda; J. Phys. Soc. Jpn. Vol. 672495 (1998).

14 M. Alzamora, M. B. Fontes, J. Larrea J, H. A. Borges, E. M. Baggio-Saitovitch and S. N. Medeiros; Phys. Rev. B 76, 125106 (2007).

15 Xiao Lin, ValentinTaufour, Sergey L. Bud'ko, Paul C. Canfield; Phys. Rev. B 88, 094405 (2013).

16 Athena, Artemis, Hephaestus: data analysis for X-ray absorption spectroscopy using IFEFFIT, J. Synchrotron Rad.12, (2005) 537.

17 J. Rodríguez-Carvajal, Physica B 192, 55 (1993); Program Fullprof, LLB-JRC, Laboratoire Léon Brillouin, CEASaclay, France, (1996). 
18 D. D. Jackson, S. K. McCall, A. B. Karki and D. P. Young; Phys. Rev. B, 76, 064408 (2007).

19 J. M. Cadogan, P. Lemoine, B. R. Slater, A. Mar and M. Avdeev, Solid State Phenom. 194, 71 (2013).

20 B. Cornut and B. Coqblin, Phys. Rev. B, 5, 4541 (1972).

${ }^{21}$ C. Kittel, Introduction to Solid State Physics, 8th edition (Wiley, New York, 2005).

22 Hiroshi Yamamoto, Masayasu Ishikawa, Katsuhiro Hasegawa and Junji Sakurai, Phys. Rev.B 5210136 (1995).

23 Z. Hossain, H. Ohmoto, K. Umeo, F. Iga, T. Suzuki, T. Takabatake, N. Takamoto and K. Kindo; Phys. Rev. B, 60,14 (1999).

24 S. K. Dhar, R. Nagarajan, Z. Hossain, E. Tominez, C. Godart, L.C. Gupta and R. Vijayaraghavan; Solid State Communications, 98, 11 (1996).

25 V. K. Anand, A. D. Hillier, D. T. Adroja, A. M. Strydom, H. Michor, K. A. McEwen and B. D. Rainford; Phys. Rev. B, 83, 064522 (2011)

26 Yuji Muro, Duhwa Eom, Naoya Takeda and Masayasu
Ishikawa ; J. Phys. Soc. Jpn. 67, 3601(1998).

27 T. Terashima, M. Kimata, S. Uji, T. Sugawara, N. Kimura, H. Aoki and H. Harima; Phys. Rev. B, 78, 205107 (2008).

28 N. Kimura, Y. Umeda, T. Asai, T. Terashima and H. Aoki; Physica B 294-295,280 (2001)

${ }^{29}$ V. T. Rajan; Phys. Rev. Lett. 51, 308 (1983).

30 A. Jesche, C. Krellner, M. de Souza, M. Lang, and C. Geibel; Phys. Rev. B, 81, 134525 (2010).

31 A Jesche, C Krellner, M de Souza, M Lang and C Geibel; New Journal of Physics 11, 103050 (2009).

32 Kazuhiko Satoh, Toshizo Fujita, Yoshiteru Maeno, Yoshichika Ōnukiand Takemi Komatsubara; J. Phys. Soc. Jpn. 58, 1012 (1989).

33 V. T. Rajan , J. H. LowensteinandN. Andrei; Phys. Rev. Lett. 49, 497 (1982)

34 V. Zlatić and R. Monnier Phys. Rev. B 71, 165109 (2005).

35 K. Miyake and H. Kohno. J. Phys. Soc. Jpn. 74, 254 (2005). 